\title{
Ventajas competitivas de las empresas cárnicas en Sinaloa
}

\section{Gildardo Ceceña Hernández*}

Competitive advantages

of the meat companies

in Sinaloa

Avantages compétitifs des entreprises de transformation de viandes animales de Sinaloa

Vantagens competitivas das empresas de carne em Sinaloa

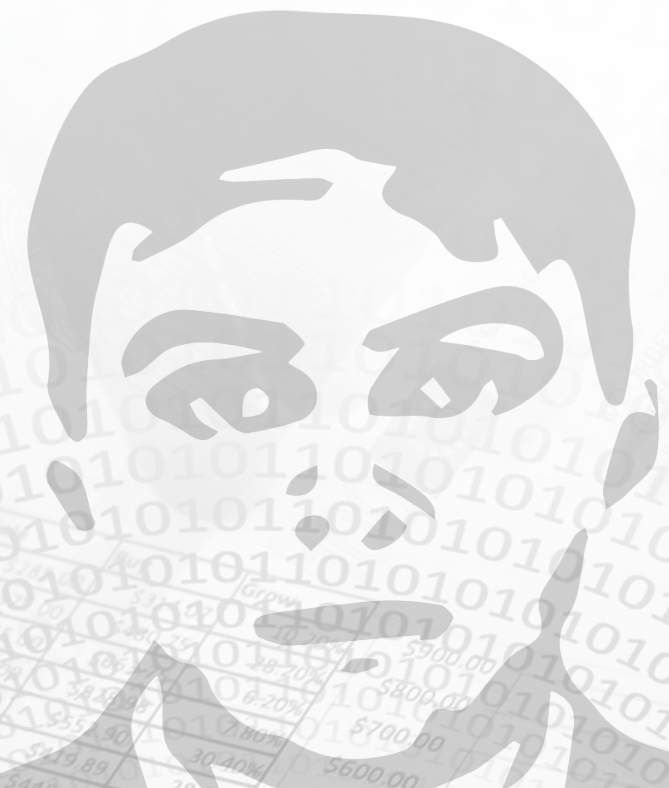

*Candidato a Doctor en Ciencias Administrativas del Instituto Politécnico Nacional-ESCA-Santo Tomás;

Mágister en Economía Regional, con énfasis en Planeación Regional y Urbana, Universidad Autónoma de Coahuila-Colegio de la Frontera Norte; Licenciado en Economía. Universidad Autónoma de Sinaloa; Docente en la Universidad Autónoma de Sinaloa, InstitutoTecnológico de Monterrey u Universidad de Durango. 


\section{RESUMEN}

El propósito de esta investigación es identificar las variables que fijan relaciones de causalidad para las ventajas competitivas de empresas del sector cárnico en la región de Sinaloa, México. El trabajo de campo se realizó a través de la aplicación de un test (24 variables) con entrevistas directas a ocho directivos empresariales, resultando como extremadamente importantes seis de las 24: satisfacción al cliente (87,5\%), factores sanitarios (75\%), calidad, producción, ventas y estandarización de procesos (62,5\%). Se concluyó que las empresas estudiadas deben implementar estrategias que les permitan tener mejores desempeños que sus competidores.

\section{ABSTRACT}

This research paper aims at identifying the variables of causal relationships for the competitive advantages of meat companies located in the region of Sinaloa, Mexico. The field study was made through the application of a test (containing 24 variables) and a short interview made to eight managers, showing the six most important out of twenty four: customer satisfaction(87.5\%), sanitary factors (75\%), quality, production, sales and standardization processes (62.5\%). We conclude that all the researched companies should implement strategies that allow them to perform better than their competitors.

\section{RESUMÉÉ}

L'objectif de cette étude est d'identifier les variables fixant les relations de causalité créant des avantages compétitifs pour les industries du secteur des viandes animales de la région mexicaine de Sinaloa. Le travail de terrain se compose de la réalisation d'un test de 24 variables dont les résultats se sont révélés extrêmement positifs. Un entretien personnalisé avec 8 dirigeants d'entreprises ( 6 des 24 variables) a également été mis en place: satisfaction client (87,5\%), facteurs sanitaires (75\%), qualité, production, ventes, standardisation des processus (62,5\%). L'analyse conclue que les entreprises étudiées doivent mettre en place des programmes de gestion stratégique leur permettant d'obtenir de meilleurs résultats que leurs concurrents.

\section{RESUMO}

O propósito desta pesquisa é identificar as variáveis que causam relações de causalidade para as vantagens competitivas de empresas do setor da carne na região de Sinaloa, México. O trabalho de campo foi feito a través da aplicação de um teste (24 variáveis) com entrevistas direitas a oito diretores empresariais, resultando extremadamente importantes seis das vinte quatro: satisfação ao cliente (87,5\%), fatores sanitários (75\%), qualidade, produção, vendas e estandardização de processos (62,5\%). Concluiu-se que as empresas estudadas devem programar estratégias que lhes permitam ter melhor desempenho do que seus concorrentes.

\section{Palabras claves}

Agroindustria Competitividad

Ventaja competitiva

Administración estratégica

Empresas cárnicas

\section{Key words}

Agroindustry Competitivenes

Competitive advantage Strategic management Meat companies.

\section{Mots clefs}

Agro-industrie

Compétitivité

Avantage comparatif

Gestion stratégique

Entreprises de viande animale.

\section{Palavras-chave}

Agroindústria

Competitividade

Vantagem competitiva Administração estratégica Empresas de carne 


\section{INTRODUCcIón}

L as ventajas competitivas pueden existir sólo cuando las empresas son capaces de producir bienes con menores costos y generar beneficios superiores que sus competidores (Porter, 1985).

Esta teoría sugiere que los Estados y las empresas deben aplicar políticas para producir mercancías de alta calidad. Se hace hincapié, en que los clientes recibirán mercancías de mayor valor en los productos que obtienen, ventaja que debe cumplirse mediante la organización de la producción al asumir costos de producción más bajos (Ranko, Berislav y Antun, 2008).

Una de las formas que actualmente utilizan las empresas para diferenciarse entre sí, es a través de la calidad del producto, la tecnología, la innovación, la marca, la reputación de la empresa y el servicio al cliente (Mose, 2010).
En este sentido el trabajo aquí presentado cuenta con cuatro apartados. El primero explora el marco teórico, dando cuenta de las contribuciones que se han hecho a través del tiempo para el concepto de ventajas competitivas, siendo una de las áreas de estudio de las ciencias administrativas. El segundo describe la metodología que se utilizó. El énfasis recae en la forma de obtener la información, así como en el procesamiento de los datos. El tercero presenta los resultados obtenidos diferenciando las principales variables que pueden ayudar a estas empresas a tener mejores rendimientos. El cuarto apartado se refiere a las conclusiones, siendo la principal contribución la determinación de variables en donde las empresas de este sector deben trabajar y aprovechar las capacidades con las que cuentan y así, poderse destacar en mercados nacionales e internacionales.

\section{REVISIÓN LITERARIA}

$\mathbf{P}$ or lo general, la ventaja competitiva es con la que una empresa destaca sobre su competidor o grupo de competidores en un mercado determinado. Fahey (1989) determina que esta ventaja puede ser cualquier elemento que distinga favorablemente a una empresa o sus productos de los competidores, desde el punto de vista de sus clientes o usuarios finales.

En este sentido, los estudios de administración han tratado el tema de la teoría de la ventaja competitiva durante muchos años. Uno de los trabajos pioneros en este campo es el de Alderson (1957), quien estableció que uno de los aspectos principales para generar ventajas, era la especia-

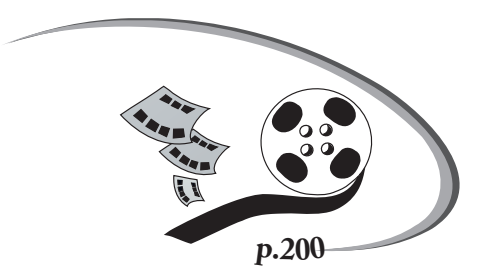

lización de los proveedores, además de sus características internas para distinguirse de los competidores.
Para la década de los ochenta, el número de trabajos en esta materia tomó un nuevo auge, pero esta vez diversificado. Por ejemplo, se identificaron tendencias como: "diferenciación" (Porter, 1985; Coyne, 1986; Hamel y Prahalad 1989); otros autores se inclinaron por "recursos únicos" (Hall, 1980; Henderson, 1983; Dierickx y Cool 1989), y otros a los aspectos clave de la ventaja competitiva, "un paso delante de los competidores" (Alderson, 1965; Ghemawat, 1986). A inicios de los noventa siguió la "visión basada en los recursos" (Barney, 1991; Conner, 1991; Peteraf, 1993) y luego los "recursos intangibles" (Hall, 1993), que se convirtieron en los temas dominantes.

Así, el cuerpo teórico relativo a las cuestiones de la empresa y su relación con el aprovechamiento óptimo de sus recursos, promovió que diversas corrientes expusieran reflexiones que conforme avanza el tiempo, exigen más la inclusión de parámetros empíricos de análisis. Barney 
(1986), en su trabajo, se inclina por la línea que retoma de forma importante el papel de la firma, los recursos y sus decisiones, en un esquema que integra estos tres elementos.

Señala que dentro del papel de la firma se encuentran aquellas fortalezas que puede usar para implementar sus estrategias; para los recursos indica procesos organizacionales, atributos, información y conocimiento, principalmente, de tal manera que estos deben ser controlados por la empresa y permitir concebir e implementar estrategias que mejoren su eficiencia y efectividad.

Siguieron investigaciones sobre aspectos específicos, por ejemplo la orientación al mercado (Hunt y Morgan, 1995), el aprendizaje organizacional (Jaworski y Kohli, 1996), la innovación (Rogers, 1995), el valor del cliente (Parasuraman, 1997) y el Marketing (Morgan y Hunt, 1994).

Posteriormente, Barney (2001) realizó un trabajo donde establece tres teorías para explicar las fuentes de ventajas competitivas. La primera, se fundamenta en la composición de los recursos de la firma; la segunda lo relaciona con la microeconomía neoclásica; y la tercera, con la economía evolutiva. Concluye que con el tiempo las empresas, mediante los recursos y estrategias adecuadas, logran mejorar su desempeño.

Para el nuevo siglo y con el fin de referenciar las ventajas competitivas, se desarrollaron otros conceptos como gestión del conocimiento, sistemas de gestión de la información, tecnología e innovación, entre otros. Flamholtz y Wei (2003) identifican lo que se denomina infraestructura organizativa (gestión de recursos, sistemas operativos, sistemas de gestión y cultura corporativa) debido a que estudiaron la pirámide del desarrollo organizacional. Kotze (2002) hace hincapié en que el valor, el tiempo, la movilidad del conocimiento, el capital intelectual, la flexibilidad y la innovación, deben ser la agenda de la competitividad.

Además, este autor identifica los temas que una empresa deberá afrontar para lograr ventajas competitivas, como por ejemplo: cultura de pensamiento estratégico, aprendizaje organizacional y trabajo en equipo. A continuación se pueden observar las contribuciones de algunos autores con referencia a las ventajas competitivas (tabla 1).

Tabla 1. Contribuciones al concepto de ventaja competitiva

\begin{tabular}{|c|c|c|}
\hline Autor / Fecha & Artículo / Título de l & Principal contribución \\
\hline $\begin{array}{l}\text { Alderson } \\
(1965)\end{array}$ & La búsqueda de la ventaja diferencial. & $\begin{array}{l}\text { Propone las bases para la ventaja diferencial: tecno- } \\
\text { lógico, jurídico y geográfico; además otra diferencia: } \\
\text { segmentación, transacción y diferenciación. }\end{array}$ \\
\hline $\begin{array}{c}\text { Hall } \\
(\mathbf{1 9 8 0})\end{array}$ & $\begin{array}{l}\text { Estrategias de supervivencia en un entor- } \\
\text { no hostil. }\end{array}$ & $\begin{array}{l}\text { El éxito empresarial se logra al costo más bajo o a la } \\
\text { mayor diferenciación. }\end{array}$ \\
\hline $\begin{array}{c}\text { Henderson } \\
\text { (1983) }\end{array}$ & La anatomía de la competencia. & $\begin{array}{l}\text { La ventaja única de la empresa sobre sus competidores } \\
\text { es adaptarse más rápido y obtener un beneficio. }\end{array}$ \\
\hline $\begin{array}{l}\text { Porter } \\
(1985)\end{array}$ & $\begin{array}{l}\text { Ventaja competitiva: creación y sosteni- } \\
\text { miento de rendimiento superior. }\end{array}$ & $\begin{array}{l}\text { Introduce la idea de la cadena de valor como la herra- } \\
\text { mienta básica para el análisis de las fuentes de ventaja } \\
\text { competitiva. }\end{array}$ \\
\hline $\begin{array}{l}\text { Coyne } \\
(1986)\end{array}$ & SCA: Qué es, o qué no es. & $\begin{array}{l}\text { Explica las condiciones necesarias para una ventaja } \\
\text { competitiva; idea de las brechas de capacidad. }\end{array}$ \\
\hline $\begin{array}{c}\text { Ghemawat } \\
\text { (1986) }\end{array}$ & Ventaja sostenible. & $\begin{array}{l}\text { Analiza las ventajas que llevan a ser sostenible: tama- } \\
\text { ño del mercado, acceso a recursos y restricciones de la } \\
\text { competencia. }\end{array}$ \\
\hline $\begin{array}{l}\text { Day and Wensley } \\
\text { (1988) }\end{array}$ & $\begin{array}{l}\text { Evaluación y ventaja: un marco para el } \\
\text { diagnóstico de superioridad competitiva. }\end{array}$ & $\begin{array}{l}\text { Las fuentes potenciales de ventaja son las habilidades } \\
\text { y recursos superiores, competidores y la perspectiva } \\
\text { del cliente. }\end{array}$ \\
\hline $\begin{array}{l}\text { Dierckx and Cool } \\
\text { (1989) }\end{array}$ & $\begin{array}{l}\text { La acumulación de activos y la sosteni- } \\
\text { bilidad de la ventaja competitiva. }\end{array}$ & $\begin{array}{l}\text { La sostenibilidad de una empresa activa se basa en la } \\
\text { facilidad con la que se puede sustituir. }\end{array}$ \\
\hline
\end{tabular}


Tabla 1. Contribuciones al concepto de ventaja competitiva (Continuación)

\begin{tabular}{|c|c|c|}
\hline $\begin{array}{l}\text { Hamel and Prahalad } \\
\text { (1989) }\end{array}$ & Intención estratégica. & $\begin{array}{l}\text { Una empresa no debe buscar una ventaja competitiva } \\
\text { sustentable, debe aprender a crear nuevas ventajas para } \\
\text { alcanzar el liderazgo mundial. }\end{array}$ \\
\hline $\begin{array}{l}\text { Barney } \\
(1991)\end{array}$ & Recursos y ventaja competitiva sostenida. & $\begin{array}{l}\text { Trata sobre cuatro indicadores de potencial de los } \\
\text { recursos de la empresa: valor, rareza, imposibilidad de } \\
\text { ser imitado y sustitución imperfecta. }\end{array}$ \\
\hline $\begin{array}{l}\text { Conner } \\
(1991)\end{array}$ & $\begin{array}{l}\text { Una comparación histórica de la teoría } \\
\text { basada en los recursos y cinco escuelas } \\
\text { de pensamiento en economía de organi- } \\
\text { zación industrial. }\end{array}$ & $\begin{array}{l}\text { Para alcanzar beneficios superiores a la media, un } \\
\text { producto de la firma debe ser diferente a los ojos de } \\
\text { los compradores. }\end{array}$ \\
\hline $\begin{array}{l}\text { Peteraf } \\
(1993)\end{array}$ & $\begin{array}{l}\text { Los pilares de la ventaja competitiva: } \\
\text { una visión basada en los recursos. }\end{array}$ & $\begin{array}{l}\text { Trata sobre cuatro condiciones para cumplir con la } \\
\text { ventaja competitiva: recursos superiores, límites de la } \\
\text { competencia, movilidad de los recursos y competencia. }\end{array}$ \\
\hline $\begin{array}{c}\text { Bharadwaj, } \\
\text { Varadajaran y Fahy } \\
(1993)\end{array}$ & $\begin{array}{l}\text { Ventaja competitiva en industrias de } \\
\text { servicios: un modelo conceptual y pro- } \\
\text { puestas de investigación. }\end{array}$ & $\begin{array}{l}\text { Evalúa la ventaja competitiva en un contexto de ser- } \\
\text { vicios de Marketing: existe sólo si es reconocido por } \\
\text { los clientes. }\end{array}$ \\
\hline $\begin{array}{c}\text { Hall } \\
(1993)\end{array}$ & $\begin{array}{l}\text { Un marco para la relación entre los recur- } \\
\text { sos intangibles y capacidades para la ven- } \\
\text { taja competitiva sostenible. }\end{array}$ & $\begin{array}{l}\text { Identifica los diferentes recursos intangibles que per- } \\
\text { miten a las empresas tener diferencias de capacidad que } \\
\text { resultan relevantes para la ventaja competitiva sostenible. }\end{array}$ \\
\hline $\begin{array}{l}\text { Day y Nedungai } \\
\text { (1994) }\end{array}$ & $\begin{array}{l}\text { Representaciones empresariales de la } \\
\text { ventaja competitiva. }\end{array}$ & $\begin{array}{l}\text { El uso de la estrategia y de la reacción con el medio } \\
\text { depende de la orientación. }\end{array}$ \\
\hline $\begin{array}{l}\text { Hunt y Morgan } \\
\text { (1995) }\end{array}$ & $\begin{array}{l}\text { La teoría de la ventaja comparativa de la } \\
\text { competencia. }\end{array}$ & $\begin{array}{l}\text { Compara la teoría neoclásica y la teoría de la ventaja } \\
\text { comparativa de la empresa. }\end{array}$ \\
\hline $\begin{array}{l}\text { Oliver } \\
(1997)\end{array}$ & $\begin{array}{l}\text { Ventaja competitiva sustentable: combi- } \\
\text { nación de visitas institucionales basados } \\
\text { en los recursos. }\end{array}$ & $\begin{array}{l}\text { Propone un modelo de heterogeneidad de las empresas, } \\
\text { lo que sugiere que tanto el capital, como los recursos } \\
\text { sean elementos necesarios para la ventaja competitiva. }\end{array}$ \\
\hline $\begin{array}{l}\text { Thomson y Coe } \\
\text { (1997) }\end{array}$ & $\begin{array}{l}\text { Ganar ventaja competitiva sustentable a } \\
\text { través de los precios. }\end{array}$ & $\begin{array}{l}\text { Propone un enfoque de fijación del valor que se puede } \\
\text { utilizar para tomar una ventaja competitiva. }\end{array}$ \\
\hline $\begin{array}{l}\text { Srivastava, Shervani y } \\
\text { Fahey } \\
\text { (1998) }\end{array}$ & $\begin{array}{l}\text { Los pilares de la ventaja competitiva: } \\
\text { una visión basada en los recursos. }\end{array}$ & $\begin{array}{l}\text { Delinea el mercado de los activos basados en dos } \\
\text { elementos principales: relacionales e intelectuales. }\end{array}$ \\
\hline $\begin{array}{l}\text { Hoffman } \\
(2000)\end{array}$ & $\begin{array}{l}\text { Ventaja competitiva en industrias de } \\
\text { servicios: un modelo conceptual y pro- } \\
\text { puestas de investigación. }\end{array}$ & $\begin{array}{l}\text { Discute el concepto de ventaja competitiva con } \\
\text { estrategia, orientación del mercado, valor del cliente } \\
\text { y Marketing. }\end{array}$ \\
\hline $\begin{array}{l}\text { Burden y Proctor } \\
\text { (2000) }\end{array}$ & $\begin{array}{l}\text { Un marco para la relación entre los } \\
\text { recursos intangibles y capacidades para } \\
\text { la ventaja competitiva sostenible. }\end{array}$ & $\begin{array}{l}\text { Satisfacer siempre las necesidades del cliente genera } \\
\text { una ventaja competitiva. }\end{array}$ \\
\hline $\begin{array}{l}\text { Sadri y Lees } \\
\quad(2001)\end{array}$ & $\begin{array}{l}\text { Representaciones empresariales de la } \\
\text { ventaja competitiva. }\end{array}$ & $\begin{array}{l}\text { La cultura positiva puede significar una ventaja com- } \\
\text { petitiva de las organizaciones contra las que se compite. }\end{array}$ \\
\hline $\begin{array}{l}\text { Coates y McDermont } \\
(2002)\end{array}$ & $\begin{array}{l}\text { La teoría de la ventaja comparativa de la } \\
\text { competencia. }\end{array}$ & $\begin{array}{l}\text { Hay diferentes categorías de competencia y las tec- } \\
\text { nológicas son fuente importante de ventaja com- } \\
\text { petitiva. }\end{array}$ \\
\hline $\begin{array}{l}\text { Adams y Lamont } \\
\text { (2003) }\end{array}$ & $\begin{array}{l}\text { Ventaja competitiva sustentable: com- } \\
\text { binación de visitas institucionales basa- } \\
\text { das en los recursos. }\end{array}$ & $\begin{array}{l}\text { La innovación organizativa y sus sistemas son el } \\
\text { factor determinante de las prácticas de innovación que } \\
\text { pueden contribuir al desarrollo de una ventaja com- } \\
\text { petitiva sostenible. }\end{array}$ \\
\hline
\end{tabular}


Tabla 1. Contribuciones al concepto de ventaja competitiva (Continuación)

\begin{tabular}{|c|l|l|}
\hline $\begin{array}{c}\text { Sharkie } \\
(\mathbf{2 0 0 3 )}\end{array}$ & $\begin{array}{l}\text { La creación de conocimiento y su lugar } \\
\text { en el desarrollo de la SCA. }\end{array}$ & $\begin{array}{l}\text { El desarrollo de una ventaja competitiva está en } \\
\text { función de la gestión y del entorno del conocimiento } \\
\text { para que la organización genere aspectos primero que } \\
\text { sus rivales. }\end{array}$ \\
\hline $\begin{array}{c}\text { Kotelnokov } \\
(\mathbf{2 0 0 4 )}\end{array}$ & $\begin{array}{l}\text { "Ventaja competitiva sostenible: ¿cómo } \\
\text { sobrevivir en contra de tu competencia } \\
\text { un largo periodo de tiempo? }\end{array}$ & $\begin{array}{l}\text { Propone un modelo de sinergia de las capacidades } \\
\text { distintivas de la empresa como fuente de ventaja } \\
\text { sostenible. }\end{array}$ \\
\hline $\begin{array}{c}\text { Khandekar y Sharma } \\
(\mathbf{2 0 0 5})\end{array}$ & $\begin{array}{l}\text { Administración de capacidades de recur- } \\
\text { sos humanos para la ventaja competitiva } \\
\text { sostenible: un análisis empírico en la } \\
\text { India de organizaciones mundiales. }\end{array}$ & $\begin{array}{l}\text { Los resultados del estudio revelan que las capacidades } \\
\text { de los recursoshumanos se correlacionan positivamente } \\
\text { con desempeño organizacional. }\end{array}$ \\
\hline
\end{tabular}

Fuente. Foon y Praven, 2010.

Siguieron aportaciones como Strandoskov (2006), quien realizó un estudio con una muestra de 133 empresas procesadoras de carne en diez países del continente europeo, encontrando una evidencia muy sólida de que las ventajas competitivas de las empresas se generaban a partir del desarrollo de nuevos productos, servicios, niveles de calidad, precios, flexibilidad tecnológica, posicionamiento de la marca y la introducción de nuevos productos.
También en un trabajo en el que se revisan las estrategias empresariales, Monge (2010) encontró que los factores calidad, precio e innovación, se pueden implementar como parte de una estrategia diferenciadora donde se canalicen los recursos y capacidades, para obtener mejores desempeños de corto y largo plazo. El factor calidad dará el soporte de una alta prestación del producto como confianza, duración como atributo principal; el precio debe estar por debajo del principal competidor y la innovación, debe llevarse a cabo en procesos y productos.

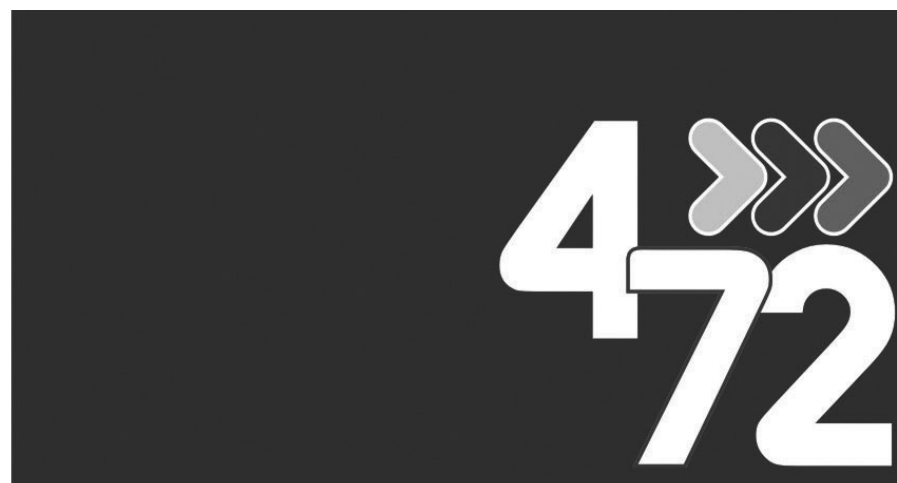

LA RED POSTAL DE COLOMBIA

w w w $4-72$. c 0 o m. c 0 


\section{METODOLOGÍA DE INVESTIGACIÓN}

E n la sección anterior se presentó la fundamentación teórica. En este apartado, se explicará la manera en que se llevó a cabo la medición de las variables de las empresas de la carne en la región de Sinaloa, para poder generar una explicación sobre la relación de estas con el sector estudiado.

La valoración de las ventajas competitivas se llevó a cabo utilizando un test de competitividad propuesto en su base por la Secretaría de Economía del gobierno mexicano; sin embargo, los autores lo modificaron para ajustarlo a este trabajo. Este pequeño test se compone de 24 variables.

El test aplicado se hizo con escala tipo Likert, en la que los valores asignados correspondían de 1 a 5 , donde 1 es no importa en lo más mínimo y 5 es extremadamente importante. Se aplicó a ocho directivos de empresas de la carne en Sinaloa que representan el $61,57 \%$ de las empresas localizadas en esta región. La información fue recabada con entrevista directa durante el mes de noviembre de 2012, en Sinaloa.

Esta metodología fue utilizada en esta investigación pues corresponde a trabajos de Benchmarking para evaluar capacidades competitivas de otros sectores económicos en México. En este sentido, es necesario comentar que se utilizó este método ya que corresponde a instrumentos aplicados en las ciencias sociales y administrativas para medir características muy diversas de fenómenos sociales en la forma más objetiva posible.

La base de este procedimiento es pedirle al sujeto que señale dentro de una serie graduada de ítems, aquellos que acepta o prefiera. Por actitud se entiende un estado de disposición psicológica adquirida a través de la experiencia que incita al individuo a reaccionar de una forma específica frente a determinados estímulos.

De tal manera que al evaluar a las empresas cárnicas de Sinaloa, buscaríamos encontrar las variables más representativas para generar algunas estrategias de mejora en ellas y acelerar el proceso competitivo. Se determinó que esto era adecuado para la investigación debido a su congruencia con la estructura presentada para el análisis de empresas en un entorno de muchas variables, en el que se incluyó el factor competitivo a través de la mejora en los procesos de la empresa, donde la pertenencia de la dotación de recursos propios promueve la competitividad a través de capacidades principales como innovación, transmisión de información efectiva, canales

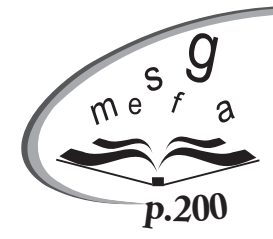
eficientes de productividad y organización. 


\section{PRINCIPALES RESULTADOS}

$\mathbf{E}$ n esta sección, se reporta la totalidad de pruebas realizadas para llegar a los resultados. Se hace mención que la primera prueba consistió en obtener el promedio ponderado de las respuestas que dieron los entrevistados. La segunda, fue obtener la media y la desviación estándar para poder definir cuáles de ellas presentan algún tipo de asociación. En la tercera prueba, efectuada a través del Software estadístico Econometrics Views, se corrieron las variables para obtener el coeficiente de correlación para cada una y poder definir cuáles son las más importantes para la toma de decisiones en el sector estudiado.

En opinión de los entrevistados, la ponderación más alta (extremadamente importante) fue alcanzada por la variable satisfacción al cliente con un $87,5 \%$, de acuerdo con el promedio ponderado. Se presentan los resultados obtenidos para cada una de las variables que se evaluaron, así como su nivel de importancia (tabla 2).

Tabla 2. Nivel de importancia de las variables competitivas

\begin{tabular}{|l|r|r|r|r|r|}
\hline \multicolumn{1}{|c|}{ Variables } & $\begin{array}{c}\mathbf{1} \\
\text { No importa en lo } \\
\text { más mínimo }\end{array}$ & $\begin{array}{c}\mathbf{2} \\
\text { Levemente } \\
\text { Importante }\end{array}$ & $\begin{array}{c}\mathbf{3} \\
\text { Importante }\end{array}$ & $\begin{array}{c}\text { M } \\
\text { Muy } \\
\text { Importante }\end{array}$ & $\begin{array}{c}\text { Extremadamente } \\
\text { Importante }\end{array}$ \\
\hline Competencia & $0(0 \%)$ & $1(12,5 \%)$ & $2(25 \%)$ & $4(50 \%)$ & $1(12,5 \%)$ \\
\hline Logística & $0(0 \%)$ & $0(0 \%)$ & $0(0 \%)$ & $6(75 \%)$ & $2(25 \%)$ \\
\hline Ventas & $0(0 \%)$ & $0(0 \%)$ & $1(12,5 \%)$ & $2(25 \%)$ & $5(62,5 \%)$ \\
\hline Producción & $0(0 \%)$ & $0(0 \%)$ & $0(0 \%)$ & $3(37,5 \%)$ & $5(62,5 \%)$ \\
\hline Capacitación empleado & $0(0 \%)$ & $0(0 \%)$ & $1(12,5 \%)$ & $3(37,5 \%)$ & $4(50 \%)$ \\
\hline Estandarización de procesos & $0(0 \%)$ & $0(0 \%)$ & $0(0 \%)$ & $3(37,5 \%)$ & $5(62,5 \%)$ \\
\hline Innovación & $0(0 \%)$ & $0(0 \%)$ & $2(25 \%)$ & $4(50 \%)$ & $2(25 \%)$ \\
\hline Marketing & $0(0 \%)$ & $2(25 \%)$ & $0(0 \%)$ & $4(50 \%)$ & $2(25 \%)$ \\
\hline Factores sanitarios & $0(0 \%)$ & $0(0 \%)$ & $0(0 \%)$ & $2(25 \%)$ & $6(75 \%)$ \\
\hline Calidad del producto & $0(0 \%)$ & $0(0 \%)$ & $1(12,5 \%)$ & $2(25 \%)$ & $5(62,5 \%)$ \\
\hline Satisfacción al cliente & $0(0 \%)$ & $0(0 \%)$ & $0(0 \%)$ & $1(12,5 \%)$ & $7(87,5 \%)$ \\
\hline Tecnología & $1(12,5 \%)$ & $0(0 \%)$ & $1(12,5 \%)$ & $3(37,5 \%)$ & $3(37,5 \%)$ \\
\hline Subsidio gubernamental & $2(25 \%)$ & $2(25 \%)$ & $3(37,5 \%)$ & $1(12,5 \%)$ & $0(0 \%)$ \\
\hline Recursos humanos & $0(0 \%)$ & $1(12,5 \%)$ & $2(25 \%)$ & $3(37,5 \%)$ & $2(25 \%)$ \\
\hline Precio & $0(0 \%)$ & $0(0 \%)$ & $3(37,5 \%)$ & $2(25 \%)$ & $3(37,5 \%)$ \\
\hline Mejora continua & $0(0 \%)$ & $0(0 \%)$ & $4(50 \%)$ & $2(25 \%)$ & $2(25 \%)$ \\
\hline Cultura corporativa & $0(0 \%)$ & $0(0 \%)$ & $4(50 \%)$ & $2(25 \%)$ & $2(25 \%)$ \\
\hline Costos de insumos & $0(0 \%)$ & $0(0 \%)$ & $2(25 \%)$ & $2(25 \%)$ & $4(50 \%)$ \\
\hline Marca & $1(12,5 \%)$ & $0(0 \%)$ & $2(25 \%)$ & $1(12,5 \%)$ & $4(50 \%)$ \\
\hline Posición del mercado & $0(0 \%)$ & $0(0 \%)$ & $0(0 \%)$ & $6(75 \%)$ & $2(25 \%)$ \\
\hline Canales de comercialización & $0(0 \%)$ & $2(25 \%)$ & $4(50 \%)$ & $2(25 \%)$ & $0(0 \%)$ \\
\hline Investigación y desarrollo & $1(12,5 \%)$ & $0(0 \%)$ & $1(12,5 \%)$ & $5(62,5 \%)$ & $1(12,5 \%)$ \\
\hline Desempeño financiero & $0(0 \%)$ & $0(0 \%)$ & $1(12,5 \%)$ & $5(62,5 \%)$ & $2(25 \%)$ \\
\hline Internacionalización & $2(25 \%)$ & $0(0 \%)$ & $2(25 \%)$ & $2(25 \%)$ & $2(25 \%)$ \\
\hline
\end{tabular}

Fuente. Elaboración propia. 
Se observa además (tabla 2), que otra de las variables con alta calificación extremadamente importante es la referente a los factores sanitarios con un $75 \%$ y para un conjunto de estas (calidad del producto, producción y ventas) se obtuvo un $62,5 \%$ de nivel de importancia.

Para la columna de muy importante las variables en orden de importancia y de acuerdo con la valoración de las entrevistas, fueron: logística y posición de mercado que obtuvieron $75 \%$; desempeño financiero e investigación y desarrollo 62,5\% e innovación y Marketing (50\%).

En este orden de ideas se obtuvo la media y desviación estándar de la medición efectuada, como se muestra en la tabla 3.

Tabla 3. Media y desviación estándar.

\begin{tabular}{|l|c|r|}
\hline \multicolumn{1}{|c|}{ Variables } & Media & $\begin{array}{c}\text { Desviación } \\
\text { estándar }\end{array}$ \\
\hline Competencia & 1,6 & 1,517 \\
\hline Logística & 1,6 & 2,608 \\
\hline Ventas & 1,6 & 2,074 \\
\hline Producción & 1,6 & 2,302 \\
\hline Capacitación empleado & 1,6 & 1,817 \\
\hline Estandarización de procesos & 1,6 & 2,302 \\
\hline Innovación & 1,6 & 1,673 \\
\hline Marketing & 1,6 & 1,673 \\
\hline Factores sanitarios & 1,6 & 2,608 \\
\hline Calidad del producto & 1,6 & 2,074 \\
\hline Satisfacción al cliente & 1,6 & 3,050 \\
\hline Tecnología & 1,6 & 1,342 \\
\hline Subsidio gubernamental & 1,6 & 1,140 \\
\hline Recursos humanos & 1,6 & 1,140 \\
\hline Precio & 1,6 & 1,517 \\
\hline Mejora continua & 1,6 & 1,673 \\
\hline Cultura corporativa & 1,6 & 1,673 \\
\hline Costos de insumos & 1,6 & 1,673 \\
\hline Marca & 1,6 & 1,517 \\
\hline Posición del mercado & 1,6 & 2,608 \\
\hline Canales de comercialización & 1,6 & 1,673 \\
\hline Investigación y desarrollo & 1,6 & 1,949 \\
\hline Desempeño financiero & 1,6 & 2,074 \\
\hline Internacionalización & 1,6 & 0,894 \\
\hline
\end{tabular}

Fuente. Elaboración propia.
De acuerdo con la información anterior, se puede constatar que en las variables de las ocho empresas se obtiene una media de 1,6. De estas variables sobresalen considerando el valor de la desviación estándar, en primer lugar, la satisfacción al cliente (3.04); en segundo lugar, factores sanitarios, logística y posición de mercados $(2,60)$; en tercero, la producción y estandarización de procesos $(2,30)$; en cuarto, desempeño financiero, calidad del producto y ventas $(2,07)$; finalmente las variables comercialización, costos, cultura corporativa, mejora continua, Marketing e innovación $(1,67)$. De las 24 variables consideradas en este estudio, estas últimas representan el 58,3\% y nos señalan de manera aislada que existe cierto tipo de asociación entre las mismas. Para ello, realizamos un ejercicio de verificación buscando comprobar si efectivamente existe algún grado de correlación.

Para realizar dicho ejercicio estadístico, se eligió como variable dependiente la de satisfacción al cliente ya que de acuerdo con los resultados de los promedios ponderados, esta obtuvo un valor de 87,5\% y una desviación estándar de 3,02 , que son los más altos. Se incorporaron aquellas variables que tuvieron un mayor nivel de asociación (figura 1).

Figura 1. Coeficiente de correlación.
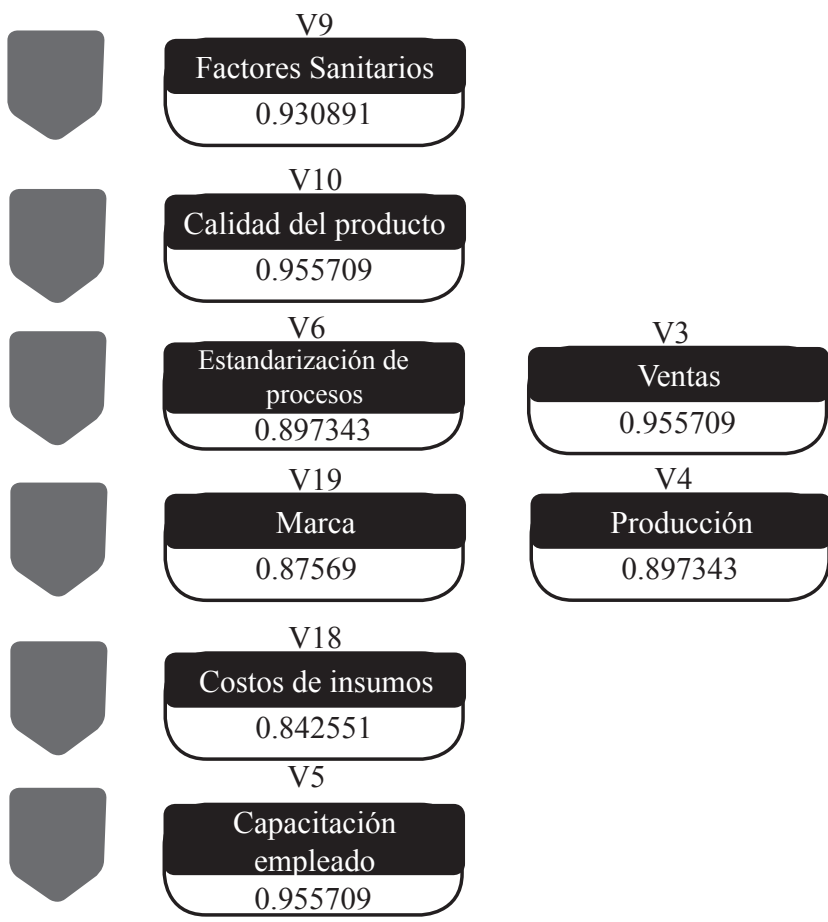

Fuente. Elaboración propia. 
De lo anterior se desprenden principalmente dos aspectos: la representatividad de las variables y el grado de asociación:

- Este grupo de variables representa un 33,3\% del conjunto (8 de 24).
- Las variables con un valor por encima del $80 \%$ son las que las empresas de la región de Sinaloa deberán atender para mejorar sus ventajas competitivas.

\section{CONCLUSIONES}

L a información presentada a partir de la investigación que se realizó en Sinaloa, muestra un entendimiento acerca de los cuales son las variables donde pueden surgir ventajas competitivas para la conducción de estrategias que les permitan a las empresas del sector cárnico tener mejores desempeños que sus competidores.

De esta manera, se contribuye al campo de estudio de la competitividad en resolver los paradigmas de cómo las empresas deben incluir en sus estrategias los recursos y capacidades como fuentes de ventajas competitivas. En esta perspectiva, se esfuerza en responder la interrogante central de cómo las empresas logran generar un rendimiento mayor a través del aprovechamiento de sus recursos y capacidades, con el fin de generar ventajas competitivas, acaparar un mayor mercado y aumentar las posibilidades de liderar su segmento económico.

Esta perspectiva infiere en participar activamente sobre el aprovechamiento de los recursos de las empresas, descartar aquellos que no son funcionales y mantener en lo posible, la ventaja competitiva sobre otras a través de la explotación de las capacidades. Esto con la finalidad de tener liderazgo en el mercado para que les dé fortaleza para competir a nivel nacional e internacional, lo que asegura de alguna manera, sus ganancias futuras y por otro lado evita depender de terceros.

Dentro de las debilidades encontradas están la disparidad de los competidores locales y la ausencia de procesos certificados destinados a fomentar la calidad. Además, no utilizan procesos de mejora continua relacionados a las formas de dirección empresarial; ejemplo de ello es que los directores generales, aun en reconocimiento de los beneficios que pudiera retribuirles fomentar medios de mejora continua, temen atender problemáticas vinculadas.

Estos resultados generan la necesidad de mejorar el gerenciamiento directivo y los procesos de innovación en las empresas, ya que Sinaloa es líder en muchos de los productos agrícolas que se ofertan en mercados nacionales e internacionales. La escasa integración productiva entre el sector extractivo de los

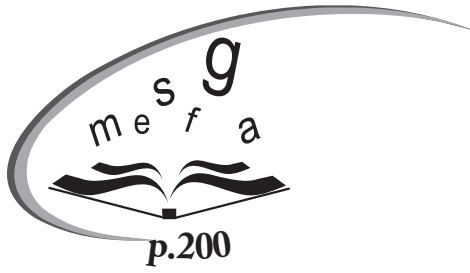
alimentos y el de industrialización, imposibilita el aumento en procesos productivos de gran alcance, que les permite abatir los rezagos mencionados y posibilita un destacado papel dentro de este sector.

Finalmente, se generan líneas futuras de trabajo e investigación y se propone la parte de comercialización como variable indispensable para aumentar el conocimiento del mercado y estilos de consumo a nivel mundial. Las grandes empresas locales deben adecuar sus procesos para lograr mantener innovación constante, con el fin de mantener la competitividad en los mercados internacionales. Además, se deben intensificar los procesos de la cadena primaria a través de la transformación de nuevos productos que satisfagan la variante demanda de los mercados nacional e internacional. Con la extensión de los procesos de comercialización a mercados extranjeros, se crea una retroalimentación que a mediano plazo retribuye a la renovación de prácticas internas y externas de la propia empresa. 
Los entrevistados señalan que el objetivo es incorporar más productos, nuevos clientes y mercados; además, que se deben desarrollar procesos tecnológicos que les permitan producir a precios bajos, de alta calidad e innovadores, ya que si no realizan estas actividades de manera estratégica, siempre tendrán el peligro latente de ser desplazados por alguno de sus competidores. Sumado a esto, la capacitación gerencial es muy necesaria para dar continuidad a las empresas en la consecución de nuevos mercados nacionales o internacionales. 


\section{REFERENCIAS}

Alderson, W. (1957). Marketing behavior and executive action. Homewood, IL: Irwin.

Alderson, W. (1965) Dynamic marketing behavior: a functionalist theory of marketing. Homewood, IL: Irwin.

Barney, J. B. (1991). Firms Resources and Sustained Competitive Advantages. Journal of Management, 17, 99-120.

Barney, J. B. (1986). Organizational culture: can it be a source of sustained competitive advantage?.Academy of Management Review, 11(3), 656-665.

Barney, J. B. (1986). Strategic factor markets: expectations, luck, and business strategy. The Institute of Management Science, 10, 1231-1241.

Barney, J. B. (2001). Resource-based theories of competitive advantage: A ten year retrospective on the resource-based view. Journal of Management, 27, 643.

Bernal García, C. E. (2010). Metodología de la Investigación. Ciudad: McGraw Hill.

Calderón, H. G. (2006). Competencias distintivas en las PyMES: Un aporte desde gestión humana. Revista de Ciencias Administrativas y Sociales, 16, 57-72.

Castañón, R. (2003). Estructura y perspectivas de la industria de alimentos en México. Revista Comercio Exterior, 114127.

Castro Monge, E. (2010). Las estrategias competitivas y su importancia en la buena gestión de las empresas. Ciencias Económicas, 247-276.

Centro de Estudios Económicos del Sector Privado (CEESP), (2005). Recuperado de http://www.ceesp.org.mx/analisise-investigacion/entorno-economico/

Collis, D. J. y Montgomery, C. A. (1995). Competing on resources: Strategy in the 1990s. Harvard Business Review, 73(4), 118-129.

Conner, K. R. (1991). An historical comparison of resource-based logic and five schools of thought within industrial organization economies: Do we have a new theory of the firm here. Journal of Management, 17, 121-154.

Coyne, K. P. (1986). Sustainable competitive advantage - what it is, what it isn't. Business Horizons, 29, 54-61. 
Day, G. S. y Wensley, R. (1988). Assessing advantage: A framework for diagnosing competitive superiority. Journal of Marketing, 52, 2-20.

Dierickx, I. y Cool, K. (1989). Asset stock accumulation and sustainability of competitive advantage. Management Science 35, 1504-1513.

Dussel Peters, M. P. (1997). Pensar globalmente y actuar regionalmente. Hacia un nuevo paradigma industrial para el siglo XXI. México: Siglo XXI y UNAM.

Fahey, L. (1989). Discovering your firm's strongest competitive advantage. In L. Fahey (ed.). Ciudad: editorial.

Fernández, E. (1996): “Factores determinantes de la competitividad empresarial”. Esic- Market, 94, 119-134.

Fernández, Z. (1993). La organización interna como ventaja competitiva para la empresa. Papeles de Economía Española, 56. 78-1993.

Flamholtz, E. y Wei, H. (2003). Searching for Competitive Advantage in the Black Box. European Management Journal, 21(2), 222-236.

Flamholtz, E. y Wei, H. (2003). The strategic planning management reader. Englewood Cliffs, NJ: Prentice Hall, pp. 1822.

Ghemawat, P. (1986). Sustainable Advantage. Harvard Business Review, 53-58.

Hall, R. (1993). A framework linking intangible resources and capabilities to sustainable competitive advantage. Strategic Management Journal, 14, 607- 618.

Hall, W. K. (1980). Survival strategies in a hostile environment. Harvard Business Review, 58, 75-85.

Hamel, G. y Prahalad, C. K. (1989). Strategic Intent. Harvard Business Review, 63-76.

Hamel, G. y Prahalad C. K. (1994). Competing for the Future. Bostón, MA, USA: Harvard Business School Press.

Henderson, B. (1983). The anatomy of competition. Journal of Marketing, 47, 7-11.

Hunt, S. D. y Morgan, R. M. (1995). “The comparative advantage theory of competition”. Journal of Marketing, 59, 1-14.

Kotelnikov, V. (2004). Sustainable competitive advantage, how to survive against your competition over a long period of time. Recuperado de http://www.1000ventures.com/business_guide/crosscuttings/sca_main.html

Kotze, J. (2002). Sustainable competitive advantage in the 21st Century. Accountancy, 11-16.

Mahoney J. y Pandian, J. R. (1992). The Resources-Based View Within the Conversation of Strategic Management. Strategic Journal, 13, 363-380.

McGrath, R. G., MacMillan, I. C. y Venkataraman, S. (1995). Defining and developing competence: A strategic process paradigm. Strategic Management Journal, 16, 251-275. 
Moses, A. (2010). Business Strategy and Competitive Advantage in Family Businesses in Ghana: The Role of Social Networking Relationships. Conference on Entrepreneurship in Africa.

Peteraf, M. A. (1993). The cornerstones of competitive advantage: A resource-based view. Strategic Management Journal, 14(3), 179-192

Pfeffer, J. (1996). Ventaja competitiva a través de la gente: cómo desencadenar el poder la fuerza de trabajo. México: CECSA.

Porter, M. (1985). Competitive Advantage: Creating and Sustaining Superior Performance. New York: Free Press.

Porter, M. (1990). The competitive advantage of nations. Harvard Busines Review, 73-93.

Porter, M. (1991). Towards a dynamic theory of strategy. Strategic Management Journal, 12, 5-29.

Porter, M. (2000). Estrategia Competitiva. Técnicas para el análisis de los sectores industriales y de la competencia. México: Grupo Editorial Patria.

Ranko, S., Berislav, B. y Antun, S. (2008). Document management system as source of competitive advantage. New Ways in Manufacturing Engineering'2008. Ciudad: editorial.

Sandoval, J. (2003). El TLCAN y la agricultura: situación general al 2002 y propuestas de solución a la problemática generada por la apertura en el 2003. México: Dirección de Servicios de Biliotecas.

Secretaría de Economía (2007). Sistema de evaluación de indicadores de competitividad. Recuperado de http://mexico. smetoolkit.org/mexico/es/content/es/2984/Sistema-de-Evaluaci\%C3\%B3n-de-Indicadores-de-CompetitividadBenchmarking-

Stinchombe, A. L. (1965). Social structure and organizations. In J.G. March (Ed.), Handbook of Organizations. Chicago: Rand.McNally, pp. 142-193.

Strandskov, J. (2006). Sources of competitive advantages and business performance. Journal of Business Economics and Management, 7(3), 119-129. 